\title{
Études historiques sur la Révolution francaise. Hommages à Victor Daline (1902-1985)
}

\section{Varoujean Pogossian}

\section{(2) OpenEdition \\ 1 Journals}

\section{Édition électronique}

URL : https://journals.openedition.org/ahrf/955

DOI : $10.4000 /$ ahrf.955

ISSN : 1952-403X

Éditeur :

Armand Colin, Société des études robespierristes

\section{Édition imprimée}

Date de publication : 1 mars 2000

Pagination : 167-170

ISSN : 0003-4436

\section{Référence électronique}

Varoujean Pogossian, «Études historiques sur la Révolution francaise. Hommages à Victor Daline (1902-1985) ", Annales historiques de la Révolution française [En ligne], 319 | janvier-mars 2000, mis en ligne le 10 avril 2006, consulté le 24 avril 2022. URL : http://journals.openedition.org/ahrf/955 ; DOI : https://doi.org/10.4000/ahrf.955

Ce document a été généré automatiquement le 24 avril 2022.

Tous droits réservés 


\title{
Études historiques sur la Révolution francaise. Hommages à Victor Daline (1902-1985)
}

\author{
Varoujean Pogossian
}

\section{RÉFÉRENCE}

Études historiques sur la Révolution francaise. Hommages à Victor Daline (1902-1985), Sous la direction d'A. TCHOUDINOV, en collaboration avec D. BOVYKINE et G.TCHERTKOVA (en russe), Moscou, 1998, $322 \mathrm{p}$.

1 Ce recueil d'articles est publié à l'occasion du 95e anniversaire de la naissance de l'éminent historien soviétique Victor Daline (1902-1995), membre du Comité directeur de la Société des études robespierristes, docteur honoris causa à l'université de Besançon.

2 Né à Odessa, Daline s'est engagé bien jeune dans le mouvement révolutionnaire. Sa jeunesse a été éclairée par les rayons du soleil de la révolution de 1917, et par conséquent, il en a subi l'empreinte ineffaçable sur sa mentalité, ses pensées et ses actions, circonstance qui se fait fortement sentir jusqu'au dernier moment de sa vie. Après avoir occupé des postes de responsable dans les organisations importantes du Parti communiste, il a abandonné en 1924 l'activité politique et à partir de ce moment a consacré toutes ses forces à l'étude de l'histoire moderne de la France.

Élève de N. Loukine, Daline a étudié dès le début de sa carrière scientifique l'histoire du mouvement socialiste en France au xxe siècle et celui de la Révolution française, ayant été l'un des premiers chercheurs marxistes de l'époque révolutionnaire. Notons qu'en dépit de l'étendue de ses intérêts scientifiques, ces deux domaines se sont constamment trouvés au centre de son attention.

4 Or, sa carrière a été brusquement interrompue en 1937. Devenu l'une des innombrables victimes de la terreur stalinienne, qui battait son plein à cette époque en URSS, il a été 
privé de la possibilité de continuer ses recherches. De son propre aveu, il a été soupçonné d'avoir des relations avec les trotskistes. La machine infernale de la terreur stalinienne l'a condamné à dix ans d'exil, alors que cette accusation n'avait été argumentée par aucune preuve. Après sa remise en liberté il a été exilé pour une deuxième fois en Sibérie en 1948 et n'est définitivement revenu à Moscou et à la science historique qu'en 1955.

5 Les travaux de Daline, écrits pendant la deuxième période de son activité scientifique, sont bien connus en France. Son capolavoro sur Babeuf a eu à Moscou deux éditions en traduction française $(1976,1987)$. Ce livre et son recueil d'articles Hommes et idées, également traduit en français (Moscou, 1983), ont été couronnés du Prix Volguine par l'Académie soviétique (1971) et ont assuré à leur auteur un succès parfaitement mérité.

6 Sa mort a été sans doute une perte douloureuse pour la science historique et surtout pour les études révolutionnaires, mais ses élèves et ses collègues continuent à Moscou, malgré la dureté des conditions apparues dans ce pays durant ces dernières années, leurs recherches dans le domaine qui était cher à Daline. Ce recueil d'articles en est la meilleure preuve.

7 Il est composé de trois parties, dont la première comprend des documents sur Daline. Dans leurs souvenirs, ses collègues (V. Smirnov et d'autres) brossent le portrait d'un homme de probité, sincère et modeste, ainsi que d'un historien dévoué, ayant été non seulement un savant remarquable, mais aussi un admirable organisateur des travaux scientifiques. Son talent d'organisateur, noté à juste titre, s'est bien démontré lors de la rédaction des Annuaires d'études françaises (31 volumes). Je me permets d'ajouter pour ma part, que ce travail pénible, qu'il réalisait avec plaisir, accaparait la majeure partie de son temps. Beaucoup d'articles sur la Révolution y ont été publiés. V.Smirnov attire l'attention sur son immense rôle dans l'amélioration et la consolidation des relations entre les historiens français et soviétiques. Les articles de nombreux historiens français et étrangers (F. Braudel, A. Soboul, J. Godechot, J. Le Goff, J.-R. Suratteau, R. Legrand, W. Markov et d'autres) y ont paru.

8 En se référant aux documents inédits tirés des archives russes, Mikhaïl Daline étudie pour la première fois un épisode important, mais inconnu à ce jour, touchant à l'activité politique de son père dans les années 1920. Il montre de la manière la plus convaincante que l'étiquette de trotskiste, attribuée à son père à la limite des années 1923-1924 lors des querelles politiques, était privée du moindre fondement. Toutefois, Daline est arrêté en 1937 et exilé « pour activité contre-révolutionnaire trotskiste » (p. 29).

9 Les éditeurs publient l'article inédit de Victor Daline sur l'éminent historien russe A. Savine (1873-1923), professeur à l'université de Moscou, ayant enseigné l'histoire moderne de l'Europe. En étudiant le journal de ce dernier, datant de 1908 à 1917, Daline a relevé avec beaucoup de finesse des renseignements intéressants sur la vie universitaire de cette époque, ainsi que des caractéristiques précises de bien des historiens russes célèbres (Klutchevski, Vipper, Kosminski, Volguine et d'autres) lancés par Savine.

10 La première partie comprend aussi la «Bibliographie des œuvres» de Daline, rédigée par l'auteur de ces lignes (pp. 70-87). Le sort de cette bibliographie, montrant les intérêts scientifiques très étendus de Daline, a été très semblable à celui de Daline. Elle a été rédigée juste après la mort de ce dernier et était destinée à être publiée dans les Annuaires d'études françaises. Mais les patrons de cette édition (Obolenskaya et d'autres), 
qui n'hésitent jamais à déclarer leurs sentiments de profonde estime à l'égard de la mémoire de Daline et saisissent toujours l'occasion, même dans ce recueil, de constater son rôle immense dans la rédaction des Annuaires, ont toutefois refusé de la publier. Ce n'est que grâce aux efforts de Tchoudinov qui, il faut le dire, n'a jamais travaillé avec Daline, qu'elle a enfin vu le jour.

Dans les deux autres parties, Tchoudinov a réuni des articles et des publications de documents présentés par les chercheurs russes et étrangers, y compris les études inédites des historiens soviétiques défunts.

E. H. Lemay suit la destinée des Constituants en analysant les causes de leur mort durant de la Révolution. Elle discute l'activité d'environ 102 députés sur 1315 de l'Assemblée constituante (soit $8 \%$ ) qui sont morts assassinés ou suicidés. Elle a dressé trois tableaux qui aident les lecteurs à mieux comprendre les motifs de leur mort, leurs orientations politiques, etc. D'après ses calculs, la majeure partie des victimes (39) étaient des nobles, 53 sur 102 ne jouèrent pas de rôle actif dans les débats politiques.

B. Baczko discute un problème compliqué, celui du rôle de Robespierre dans la Terreur. Ce thème, comme le note l'auteur, est depuis longtemps devenu «classique» pour l'historiographie révolutionnaire. N'ayant pas utilisé de documents nouveaux, l'auteur se limite à discuter quelques aspects importants, ceux de l'attitude de Robespierre à l'égard de la Terreur, ses relations avec le peuple, ainsi que l'influence laissée sur lui-même par sa propre activité politique et étatique. D'abord, il constate que la Terreur n'a pas été réalisée d'après des projets préparés préalablement. Il croit que l'un des discours politiques de Robespierre, prononcé à l'automne 1792, a servi de point de départ pour l'installation du monopole de l'État révolutionnaire. Robespierre avait légitimisé et systématisé la politique de la Terreur, notamment en l'an II. En même temps, il affirme, qu'étant un homme d'État purement parlementaire, Robespierre n'était pas au courant des dures réalités de la Terreur. Il voulait simplement voir une Terreur pure, comme était pur le peuple. Mais Baczko évite d'expliquer ce qu'avait en vue Robespierre sous le terme de "peuple»?

Quelques problèmes de l'histoire de l'an II sont étudiés dans les trois fragments inédits du regretté professeur Y. Zakher (1893-1963), publiés par A. Gordon. Ils concernent notamment Jacques Roux et sont d'un grand intérêt surtout pour les historiens russes, d'autant plus si l'on prend en considération les travaux déjà publiés du regretté W. Markov.

15 L'historien anglais A. Forrest évoque un problème intéressant, celui du rôle des soldats français dans la diffusion de la Révolution en Europe. Il considère les soldats comme les «premiers missionnaires de la France révolutionnaire». Quant à l'armée, il considère, non sans raison, que celle-ci était, même sous le Directoire, très politisée. Cependant en dépit de ces circonstances, la politique de la France révolutionnaire dans les pays conquis a fait naître des résistances, car le trait essentiel de l'occupation française était devenu la spoliation. Donc, l'une des conséquences de ces conquêtes a été, d'après Forrest, l'apparition du nationalisme en Europe, devenu au xixe siècle l'une des traces les plus marquantes laissées par la Révolution française (p. 177).

16 L'adoption de la Déclaration des droits en l'an III et les discussions auxquelles elle a donné lieu sont analysées par Y. Bosc.

17 E. Lébédéva publie un autographe inédit d'Augustin Robespierre daté du 31 octobre 1793, quand il se trouvait à Nice. Ce document, tiré des archives russes, touche les 
problèmes quotidiens de l'armée française. Par ailleurs, Y. Danilova présente deux pages sorties de la plume de l'éminent historien russe N. Karcev (1850-1931) sur Saint-Just.

L'histoire de la ligne descendante de la Révolution demeure toujours le talon d'Achille de l'historiographie révolutionnaire russe. Dans ce contexte, il nous faut citer l'intérêt croissant à l'égard de l'époque thermidorienne. Le thème choisi par D. Bovykine (Le pouvoir exécutif dans la Constitution de l'an III de la République) est neuf pour l'historiographie soviétique/russe. La première partie de l'article, dans laquelle il discute les différentes propositions touchant les questions constitutionnelles posées lors de la préparation de la Constitution, est plus intéressante, car elle se base sur les documents des Archives nationales. En se référant également aux sources publiées (les articles de presse, etc.), l'auteur analyse aussi le problème de la séparation des pouvoirs réalisée dans la Constitution de l'an III et le rôle dévolu au futur pouvoir exécutif. Le Directoire n'est donc devenu, de son avis, que l'exécuteur de la volonté du Corps législatif qui a résolu son sort. Ces conclusions essentielles se joignent à celles de $\mathrm{M}$. Troper, spécialiste en droit français.

19 L'historien de Babeuf G. Tchertkova publie une lettre inédite d'un auteur inconnu qui est datée du 13 vendémiaire an III et adressée au Tribun du peuple. Celle-ci, tirée des archives personnelles de Babeuf, nous aide à mieux comprendre l'ambiance compliquée régnant au sein de la Convention après le 9 Thermidor.

Nous saluons la publication de l'article du regretté professeur K. Dobrolioubski (1885-1953), auteur de nombreux travaux de valeur sur l'époque thermidorienne, préparé à la publication par B. Kaganovitch, sur le Théâtre à l'époque thermidorienne. C'est une étude minutieuse sur les spectacles de cette époque. L'auteur constate qu'après le 9 Thermidor le répertoire théâtral répondait aux exigences urgentes de la vie: la persécution des Jacobins, la défense de la République contre la menace de la coalition monarchique et d'autres sujets se trouvant au centre de l'attention des nouveaux maîtres de la France et donc reflétés dans le répertoire théâtral.

21 Dans un article théorique «La Grande Révolution française comme phénomène de la culture russe» A. Gordon analyse l'image de la Révolution dans l'esprit public russe au xixe siècle, l'intérêt qu'elle avait suscité à l'époque soviétique, et constate que la Révolution française est devenue une référence dans la culture russe. D'après lui, l'image de la Révolution «vit conformément aux lois de la vie russe, meurt et ressuscite conformément aux exigences de la conscience publique du pays, s'intègre aux processus profonds du développement national» (p.245).

Quelques articles portent sur des sujets historiographiques. Celui d'Anatoli Ado (1928-1995), «La Révolution française dans l'historiographie soviétique», est rédigé en 1990. Bien que ce sujet soit étudié en maintes occasions par des chercheurs divers (V.Daline, V. Dounaevski et d'autres), son approche est toutefois originale. Ce n'est pas un aperçu historiographique de la littérature soviétique, mais une étude analytique dans laquelle il a noté en premier lieu les particularités de l'historiographie soviétique de la Révolution: la dominance sur la science historique de la lutte idéologique et politique, l'étude de la Révolution quasi-exclusivement d'en bas, la concentration de l'attention principalement sur l'époque de la dictature jacobine, etc. En outre, les conditions historiques ont contraint les historiens soviétiques à élaborer une conception unique de la Révolution, qui a dominé jusqu'aux années 1980. Toutes ces circonstances ont empêché, à son avis, de présenter l'image de la Révolution dans toute 
sa diversité. A. Ado a remarqué aussi les nouvelles tendances apparues en ex-URSS à la fin des années 1980 (l'étude de la position de la noblesse et de la bourgeoisie, celle de l'activité des forces politiques de droite, etc.). Citons obligatoirement les mérites du regretté savant dans l'organisation de l'étude de l'époque révolutionnaire dans toute sa diversité.

L'article historiographique de Tchoudinov est consacré à un autre sujet. Il a suivi le destin des archives de G. Romme et a analysé en profondeur toute la littérature historique existant sur l'activité de celui-ci et de son élève P. Stroganoff. En somme, il a conclu que l'histoire des relations du «prince russe» et du «régicide français» attend encore son chercheur. Afin d'atteindre ce but, il est nécessaire, comme il l'affirme, d'utiliser les documents des archives russes.

D. Rostislavlev discute la conception de la dictature jacobine avancée par Karcev. Parmi les nombreuses observations de l'auteur, citons celle d'après laquelle Karcev, en formulant sa conception, a examiné les différentes notions du processus historique. Il a notamment pris en considération la rupture historique, ainsi que les «relations entre les institutions de l'Ancien Régime et de la Révolution» (p. 168).

25 A. Tyrsenko présente l'une des éditions françaises plus ou moins récentes, celle du Dictionnaire des Constituants, 1789-1791 (Paris, 1991) publié sous la direction d'E.H. Lemay et constate qu'il est un utile instrument de travail.

La publication de ce recueil prouve une profonde estime à l'égard de la mémoire de Victor Daline. En même temps, il est un exemple de fructueuse coopération internationale. 